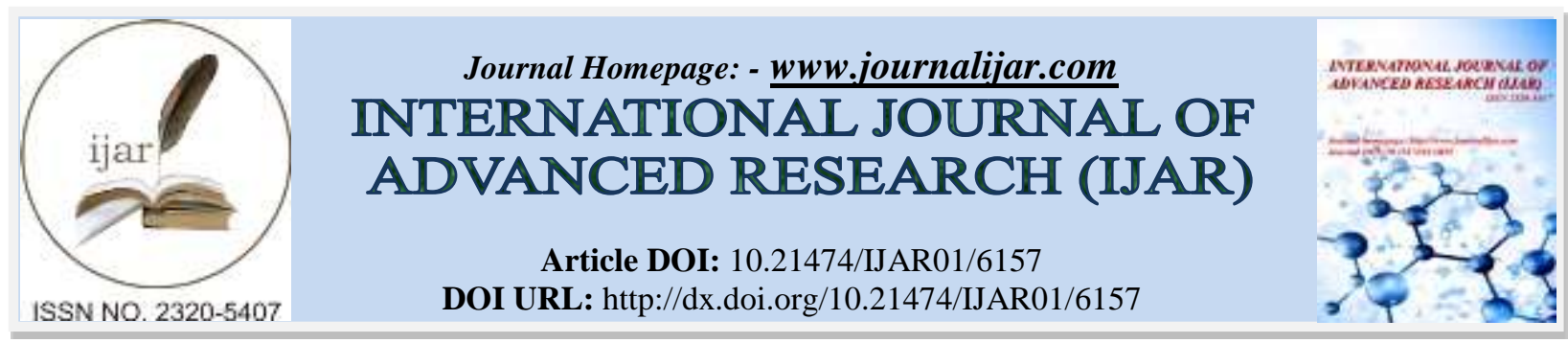

RESEARCH ARTICLE

\title{
THE IMPACT OF MUTATION AND PROMOTION ON THE PERFORMANCE OF SOUTH KALIMANTAN GOVERNMENT'S EMPLOYEES.
}

Muhammad berty nakir.

Study Program of Human Resource Development, Postgraduate Program Universitas Airlangga.

\section{Manuscript Info}

Manuscript History

Received: 24 October 2017

Final Accepted: 26 November 2017

Published: December 2017

Key words:-

Mutation, Promotion, Employees Performance.

\section{Abstract}

The purposes of this study was to understand the impact of mutation and promotion of south kalimantan government on their employees performance. Variables which related to the study were mutation (X1), promotion (X2), and the employees' performance (Y). The type of data used in this study consist of primary data which directly obtained from the respondent through questionaire, interviews, observation, and secondary data which related to the observed object and were already prepared by other parties. As much as 44 samples were taken to be observed. According to $\mathrm{t}$ test for mutation (variable $\mathrm{X} 1$ ), it is discovered that this variable has significancy value less than $t$ table value $(2,0195)$, which is $(-0,328<\mathrm{t}$ table $)$ and can be concluded that mutation (X1) has no significant impact to the employees' performance. Whereas, promotion as (variable X2) has significancy value over $t$ table $(2,0195)$ which is 3,948 . It can be concluded that promotion (X2) was significantly affect the performance of south kalimantan government employees.

Copy Right, IJAR, 2017,. All rights reserved.

\section{Background:-}

According to Undang-Undang Republik Indonesia Nomor 5 Tahun 2014, aparatur sipil negara is a proffesion or human resources which has agreement to work for government institution and being inaugurated by employment functionary. They were given a job and paid under the national regulations

The continuity of a management system at the government of south kalimantan province was very affected by both quality and quantity of its human resources. The handling of human resources was different to other production factor because it is always developed and getting better in both its quality and quantity. human resources management was required in order to make sure the continuity of an organization so that human resources utilization fit to its requirements

In order to achieve such high performance, the government of south kalimantan province has to pay attention on everything that is related to performance improvement. One of many factors that promotes performance improvement were mutation and promotion. Mutation was believed to give a whole different working situation so that saturation on the employees can be avoided and improvement on their performance can be expected. Good performance will get the employees closer to promotion, thus they will be motivated to keep their good work in place. Considering all those things, a study related to mutation and promotion was being done to get to know their impact on the performance of south kalimantan governement's employees. 


\section{Objectives:-}

The objectives of this study was to understand the impact of both mutation and promotion on government employees' performance in the province of south kalimantan.

\section{Conceptual Framework And Hypothesis:- \\ Conceptual Framework:-}

$$
\text { Variabel Independen }
$$

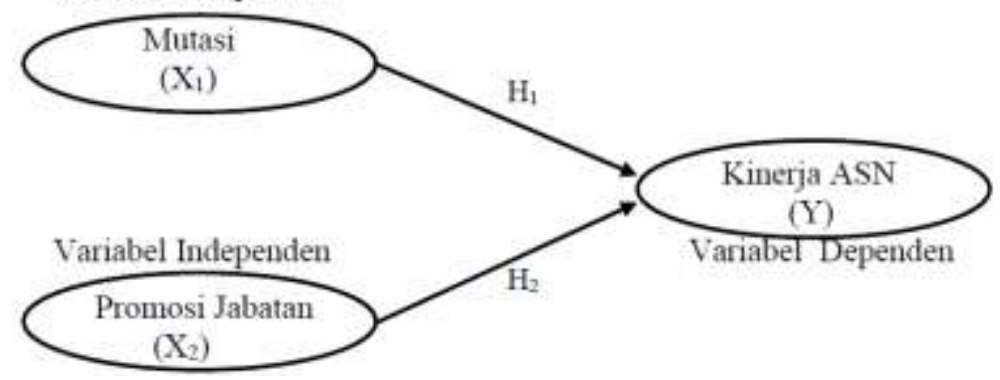

Gambar 1:- Conceptual Framework

$\mathrm{X}_{1} \quad$ : Mutation (variabel bebas/independen)

$\mathrm{X}_{2} \quad$ : Promotion (variabel bebas/independen)

Y : Employees Performance (variabel terikat/dependen)

\section{Hypothesis:-}

Hypothesis of this study were as follows:

$\mathrm{H}_{1} \quad$ : the impact of mutation.

$\mathrm{H}_{2}$ : the impact of promotion.

\section{Research Methodology:-}

The Type and Source of Data:-

The type of this study was survey which data has taken from employment agency of south kalimantan province. Two type of data used in this study were primary which directly acquired from the study object and secondary which obtained undirectly through result of another study, books, articles, other kind of publications, and also related institution that relevant to the topic.

\section{Sample and Population:-}

Population in this study was government employees who has been mutated, promoted and being mutated and promoted at once. Slovin formula was used to determine the number of sample (Sugiyono, 2010). The number of population and deviation were 78 person and $10 \%$ respectively. The number of sample was formulated as follow:

$$
\begin{aligned}
n & =\frac{N}{1+n(e)^{2}} \\
n & =\frac{78}{1+78(0,1)^{2}} \\
n & =43,82(44)
\end{aligned}
$$

Keterangan:

$$
\begin{aligned}
& \mathrm{n}=\text { Number of sample } \\
& \mathrm{N}=\text { Number of population } \\
& e=\text { error tolerance }
\end{aligned}
$$

\section{Definisi Operasional Variabel:-}

Mutation (X1) is a process of functionality, responsibility and status adjustment for one in order to obtain satisfaction and achievement as much as possible to the country, whereas Promotion (X2) is a transition of employees from one position to another which status and responsibility became higher as a consequence. According to Hasibuan (2011), promotion is a transition where authority and responsibility within an organization became higher, thus title, status and income were also increased. 
Employees performance is the result of one in doing the job which consist of planning, investigation, coordination, evaluation, control, staff recruitment, negotiation, representation, and other that given.

Tabel 1:- Study variable and dimension

\begin{tabular}{|l|l|l|}
\hline \multicolumn{1}{|c|}{ Variabel of study } & \multicolumn{1}{|c|}{ Definition } & \multicolumn{1}{c|}{ Dimension } \\
\hline Mutation $\left(\mathrm{X}_{1}\right)$ & $\begin{array}{l}\text { process of functionality, responsibility and status } \\
\text { adjustment for one in order to obtain satisfaction and } \\
\text { achievement as much as possible to the country. }\end{array}$ & $\begin{array}{l}\text { 1. Frequency } \\
\text { 2. Reason } \\
\text { 3. Transparency } \\
\text { 4. Accuracy }\end{array}$ \\
\hline Promotion $\left(\mathrm{X}_{2}\right)$ & $\begin{array}{l}\text { transition of employees from one position to another } \\
\text { which status and responsibility became higher as a } \\
\text { consequence. }\end{array}$ & $\begin{array}{l}\text { 1. Government } \\
\text { management policy }\end{array}$ \\
2. Criteria
\end{tabular}

Analysis Methods:-

Source: Analysis result (2017)

The tools in this study were as follow:

1. Instrument Validity Test :-

According to Jogiyanto (2013), validity was defined as a degree of tool's precision on measured or observed object. Value of validity exhibit how much deviation occurred on each variable. Validity can be done by corelating each item score to all item being questioned. Minimum limit that can be seen as valid was if $r$ table $>r$ analyzed. $R$ table was obtained from total respondent.

\section{Instrument Reliability Test:-}

According to Jogiyanto (2013), reliability is a degree of accuracy which exhibited by the instrument where test can be done internally on item's consistency analysis. Ghozali (2011) explained, variable is reliable if Cronbach Alpha value $>0,6$.

\section{Classic Assumption Test:-}

\section{Normality Test:-}

Normality test was purposedly test the distribution regression model of data which verge to normal distribution. This statistical test become invalid if this assumption being violated (Ghozali, 2011).

\section{Uji Heteroskedastisitas:-}

Uji heterokedastisitas was purposedly done to test the regression model so that inequality between observations could be known. If residual variant between them was fixed, it is defined as homoskedastisitas. heterokedastisitas test was undergone with statistical gletser test. This method is being done by regressing residual absolute value against independent variable. If there is no independent variable that has significant impact to residual absolute value, there is also no heterokedastisitas (Ghozali, 2011).

\section{Multicolinearity Test:-}

Multicolinearity test was purposedly being done to find out the corelation between independent variables within regression model by looking at the tolerance or variance inflation factor (VIF), where if it was higher or less than $10 \%$, there is no multicolinearity (Ghozali, 2011).

\section{Multiple Linear Regression Analysis Test:-}

This study use multiple regression with Moderated Regression Analysis (MRA) to obtain the impact of each variable on ASN performance as independent variable. To achieve not more than 5\% of error, SPSS 2.2 was utilized to test the hypothesis. Regression model used to test the hypothesis is as follow:

$$
\mathbf{Y}=\alpha+\beta_{1} \mathbf{X}_{1}+\beta_{2} \mathbf{X}_{2}+\mathbf{e}
$$

$\mathrm{Y}=$ Performance

$\alpha=$ constanta 


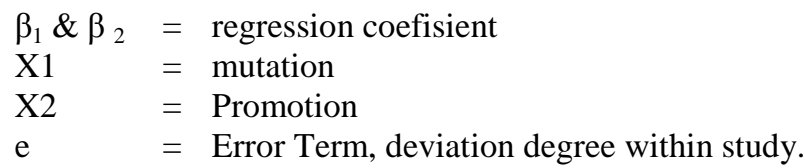

\section{Result And Discussion:-}

Instrumental Validity Test:-

Respondent in this study was 44 person, thus $r$ table obtained was 0,297 . The result of the three variable test exhibit that all collected data were valid as the Corrected Item-Total Correlation value was higher than $r$ table which is 0,297 . It indicates that all items on each variable concept were appropriate to be used as measurement.

Tabel 2:- Instrumental validity test result

\begin{tabular}{|c|c|c|c|c|}
\hline Variable & Question items & $\begin{array}{l}\text { Corrected Item- } \\
\text { Total Correlation }\end{array}$ & $r$ table value & indication \\
\hline \multirow[t]{12}{*}{ Mutation } & P1 & 0,678 & 0,297 & Valid \\
\hline & P2 & 0,442 & 0,297 & Valid \\
\hline & P3 & 0,452 & 0,297 & Valid \\
\hline & P4 & 0,444 & 0,297 & Valid \\
\hline & P5 & 0,721 & 0,297 & Valid \\
\hline & P6 & 0,471 & 0,297 & Valid \\
\hline & P7 & 0,486 & 0,297 & Valid \\
\hline & P8 & 0,222 & 0,297 & Valid \\
\hline & P9 & 0,517 & 0,297 & Valid \\
\hline & P10 & 0,636 & 0,297 & Valid \\
\hline & $\mathrm{P} 11$ & 0,429 & 0,297 & Valid \\
\hline & $\mathrm{P} 12$ & 0,501 & 0,297 & Valid \\
\hline Variable & Question items & $\begin{array}{c}\text { Corrected Item- } \\
\text { Total Correlation }\end{array}$ & r table value & indication \\
\hline \multirow[t]{12}{*}{ Promotion } & P1 & 0,640 & 0,297 & Valid \\
\hline & P2 & 0,588 & 0,297 & Valid \\
\hline & P3 & 0,606 & 0,297 & Valid \\
\hline & P4 & 0,364 & 0,297 & Valid \\
\hline & P5 & 0,536 & 0,297 & Valid \\
\hline & P6 & 0,709 & 0,297 & Valid \\
\hline & P7 & 0,317 & 0,297 & Valid \\
\hline & P8 & 0,835 & 0,297 & Valid \\
\hline & P9 & 0,777 & 0,297 & Valid \\
\hline & P10 & 0,813 & 0,297 & Valid \\
\hline & P11 & 0,533 & 0,297 & Valid \\
\hline & P12 & 0,408 & 0,297 & Valid \\
\hline \multirow[t]{12}{*}{ Performance } & $\mathrm{P} 1$ & 0,674 & 0,297 & Valid \\
\hline & $\mathrm{P} 2$ & 0,857 & 0,297 & Valid \\
\hline & P3 & 0,905 & 0,297 & Valid \\
\hline & $\mathrm{P} 4$ & 0,785 & 0,297 & Valid \\
\hline & P5 & 0,877 & 0,297 & Valid \\
\hline & $\mathrm{P} 6$ & 0,892 & 0,297 & Valid \\
\hline & $\mathrm{P} 7$ & 0,935 & 0,297 & Valid \\
\hline & P8 & 0,910 & 0,297 & Valid \\
\hline & $\mathrm{P} 9$ & 0,926 & 0,297 & Valid \\
\hline & $\mathrm{P} 10$ & 0,846 & 0,297 & Valid \\
\hline & P11 & 0,896 & 0,297 & Valid \\
\hline & $\mathrm{P} 12$ & 0,886 & 0,297 & Valid \\
\hline
\end{tabular}




\section{Instrument Reability Test:-}

Reliability test was using Cronbach's Alpha. The result will be compared to minimum acceptable realibility coefisient. if Cronbach's Alpha value was higher than 0,6, then the instrument is reliable. However, if it was less than 0,6 , then it is not reliable. The result of reliability test using SPSS were as follow:

Tabel 3:- Instrumental Reability Test Result

\begin{tabular}{|l|c|c|}
\hline \multicolumn{1}{|c|}{ Variable } & Cronbach's Alpha Analyzed & Indication \\
\hline Mutation $\left(\mathrm{X}_{1}\right)$ & 0,723 & Reliable \\
\hline Promotion $\left(\mathrm{X}_{2}\right)$ & 0,751 & Reliable \\
\hline Performance $(\mathrm{Y})$ & 0,782 & Reliable \\
\hline
\end{tabular}

Source: SPSS Analysis (2017)

According to the analysis result using SPSS program, it is indicated that croanbach alpha value of mutation (variable $\mathrm{X} 1$ ), promotion (variable X2), and performance were all higher than 0,6 , thus the respondent's answer was reliable.

\section{Classic Assumption Test:-}

Normality test was purposedly done to find abnormal distribution of disturbance or residual variable within the regression model. If this assumption being violated, the statistical test become invalid for small number of sample (Ghozali, 2011). Normality test can be seen at table 3 .

Tabel 4:- Kolmogrov-Smirnov Normality Test

\begin{tabular}{|c|c|c|}
\hline \multicolumn{3}{|c|}{ One-Sample Kolmogorov-Smirnov Test } \\
\hline & & Unstandardized Residual \\
\hline \multicolumn{2}{|l|}{$\mathrm{N}$} & 44 \\
\hline \multirow[t]{2}{*}{ Normal Parameters ${ }^{\mathrm{a}, \mathrm{b}}$} & Mean & .0000000 \\
\hline & Std. Deviation & 7.42459996 \\
\hline \multirow[t]{3}{*}{ Most Extreme Differences } & Absolute & .070 \\
\hline & Positive & .048 \\
\hline & Negative & -.070 \\
\hline \multicolumn{2}{|l|}{ Statistic Test } & .070 \\
\hline \multicolumn{2}{|l|}{ Asymp. Sig. (2-tailed) } & $200^{\mathrm{c,d}}$ \\
\hline \multicolumn{3}{|l|}{ a. Test distribution is Normal. } \\
\hline \multicolumn{3}{|c|}{ b. Calculated from data. } \\
\hline \multicolumn{3}{|c|}{ c. Lilliefors Significance Correction. } \\
\hline
\end{tabular}

Source: SPSS Analysis (2017)

According to Kolmogrov-Smirnov Normality Test shown on table 4, asymp.sig value was 0,200 which higher than significancy value $(0,05)$. Considering the condition above, residual variable was normally distributed.

Uji heteroskedastisitas purposedly done to test the variance discontinuity of residual between observations within the regression model. Result of uji heteroskedastisitas using SPSS shown on the table 5 below.

Tabel 5:- Uji Heteroskedastisitas

\begin{tabular}{|c|c|c|c|c|c|c|}
\hline \multicolumn{7}{|c|}{ Coefficients $^{\mathrm{a}}$} \\
\hline \multirow{2}{*}{\multicolumn{2}{|c|}{ Model }} & \multicolumn{2}{|c|}{ Unstandardized Coefficients } & \multirow{2}{*}{$\begin{array}{c}\text { Standardized } \\
\text { Coefficients } \\
\text { Beta } \\
\end{array}$} & \multirow[t]{2}{*}{$\mathrm{t}$} & \multirow[t]{2}{*}{ Sig. } \\
\hline & & B & Std. Error & & & \\
\hline \multirow[t]{3}{*}{1} & (Constant) & 22.254 & 3.587 & & 6.205 & .000 \\
\hline & Mutation & -.231 & .138 & -.323 & -1.672 & .102 \\
\hline & Promotion & -.203 & .128 & -.304 & -1.577 & .122 \\
\hline
\end{tabular}

Source: SPSS analysis (2017)

There is no independent variable which significantly affect the fixed one as the probability of independent variable which consist of mutation and promotion were 0,102 and 0,122 respectively, above the significancy value of 5\%. Jadi dapat dinyatakan bahwa model regresi tidak mengarah adanya heteroskedastisitas, sehingga layak untuk digunakan. 
According to Puspitasari \& Santoso (2013), multicolinearity test was purposedly done to find the corelation between independent variables within regression model. Statistical identification to find multicolinearity can be done by calculating variance inflation factor (VIF). Multicolinearity test result using SPSS can be seen on table 6 below.

Tabel 6:- Multicolinearity test

\begin{tabular}{|c|c|c|c|c|c|c|c|c|}
\hline \multicolumn{9}{|c|}{ Coefficients $^{\mathrm{a}}$} \\
\hline \multirow{2}{*}{\multicolumn{2}{|c|}{ Model }} & \multicolumn{2}{|c|}{$\begin{array}{l}\text { Unstandardized } \\
\text { Coefficients }\end{array}$} & \multirow{2}{*}{$\begin{array}{c}\begin{array}{l}\text { Standardized } \\
\text { Coefficients }\end{array} \\
\text { Beta }\end{array}$} & \multirow[t]{2}{*}{$\mathrm{t}$} & \multirow[t]{2}{*}{ Sig. } & \multicolumn{2}{|c|}{ Collinearity Statistics } \\
\hline & & $\mathrm{B}$ & $\begin{array}{l}\text { Std. } \\
\text { Error }\end{array}$ & & & & Tolerance & VIF \\
\hline \multirow[t]{3}{*}{1} & (Constant) & 10.288 & 6.807 & & 1.511 & .138 & & \\
\hline & Mutation & -.086 & .262 & -.059 & -.328 & .745 & .429 & 2.330 \\
\hline & Promotion & .962 & .244 & .705 & 3.947 & .000 & .429 & 2.330 \\
\hline
\end{tabular}

Source: SPSS analysis (2017)

According to table 6 , VIF value of mutation (X1) and promotion (X2) were $(2,330<5)$ and $(2,330<5)$ respectively, which were less than 5 and indicates no multicolinearity occurred between independent variables within regression model. This also happened in tolerance value of each variable which are as much as 0,429 , more than 0,1 .

\section{Multiple Linear Regression Analysis Test:-}

Regression model calculation result on SPSS program is shown on table 7 below:

Tabel 7:- Uji Serempak (Uji-F)

\begin{tabular}{|c|c|c|c|c|c|c|}
\hline \multicolumn{7}{|c|}{ ANOVA $^{\mathrm{a}}$} \\
\hline \multicolumn{2}{|c|}{ Model } & Sum of Squares & df & Mean Square & $\mathrm{F}$ & Sig. \\
\hline \multirow[t]{3}{*}{1} & Regression & 2049.904 & 2 & 1024.952 & 15.996 & $.000^{\mathrm{b}}$ \\
\hline & Residual & 2627.073 & 41 & 64.075 & & \\
\hline & Total & 4676.977 & 43 & & & \\
\hline
\end{tabular}

Source: SPSS analysis (2017)

Based on ANOVA, F number was 15,996 with Sig 0,000. Total sample was 44 with variables (X1 and X2) in which on the $\mathrm{F}$ table df 1 and df 2 was 2 and 42 respectively, $F$ table value $=3,22$. F number value that is higher than $F$ table $(15,996>3,11)$ with Sig value 0,000 , which smaller than alpha $(0,000<0,05)$, indicates that each variable simultaneously give significant impact on employees (ASN) performance.

According to table 8 , regression equation was $\mathrm{Y}=10,288-0,86 \mathrm{X}_{1}+0,962 \mathrm{X}_{2}$. T table value from 44 samples with 2 variables (X1 and X2) was 2,0195.

Tabel 8:- Partial test

\begin{tabular}{|c|c|c|c|c|c|c|}
\hline & & & Coefficients $^{2}$ & & & \\
\hline & & Unstandardiz & oefficients & Standardized & $\mathrm{t}$ & Sig. \\
\hline & & B & Std. Error & Beta & & \\
\hline 1 & (Constant) & 10.288 & 6.807 & & 1.511 & .138 \\
\hline & Mutation & -.086 & .262 & -.059 & -.328 & .745 \\
\hline & Promotion & .962 & .244 & .705 & 3.947 & .000 \\
\hline
\end{tabular}

Source: SPSS Analysis (2017)

According to table 8 , mutation (X1) has no significant impact to ASN performance with significancy value of 0,745 $>0,05$ and $\mathrm{T}$ number of $-0,328<\mathrm{T}$ table, whereas promotion (X2) indicates an opposite impact with significancy value and $t$ number of $0,000<0,05$ and 3,948 $>t$ table respectively.

Determination coeficient test result is shown on table 9 below. 
Tabel 9:- Determination coeficient test result $\left(\mathrm{R}^{2}\right)$

\begin{tabular}{|c|c|c|c|c|}
\hline \multicolumn{5}{|c|}{ Model Summary } \\
\hline Model & $\mathrm{R}$ & R Square & Adjusted R Square & Std. Error of the Estimate \\
\hline 1 & $.662^{\mathrm{a}}$ & .438 & .411 & 8.00468 \\
\hline
\end{tabular}

Source: SPSS analysis (2017)

Determination coeficient $\left(\mathrm{R}^{2}\right)$ measures the ability of model in defining dependent variable variation. A small R2 value indicates that independent variables on defining dependent one was very limited (Puspitasari \& Santoso, 2013). Determination coeficient value (Adjusted $R$ Square) was 0,411 . It indicates that mutation and promotion affect ASN performance as much as $41,1 \%$, whereas $58,9 \%$ was affected by other variable outside this regression model.

\section{Conclusion:-}

According to the analysis result and discussion on the impact of mutation and promotion to south kalimantan government employees' performance, the conclusions were as follow:

1. On the $\mathrm{t}$ test for mutation (variable $\mathrm{X} 1$ ), it is known that this variable has significancy value less than $\mathrm{t}$ table $(2,0195)$ which as much as $-0,328<t$ table, therefore has no significant impact on the employees' performance.

2. On the $\mathrm{t}$ test for promotion (variable $\mathrm{X} 2$ ), it is known that this variable has significancy value over $\mathrm{t}$ table $(2,0195)$ which as much as $3,948>t$ table, therefore has a significant impact on the employees' performance.

\section{Reference:-}

1. Ghozali, Imam, 2011. Aplikasi Analisis Multivariate Dengan Program SPSS. Badan Penerbit Universitas Diponegoro. Semarang.

2. Hasibuan, Malayu S.P., 2011. Manajemen Sumber Daya Manusia. PT Bumi Aksara. Jakarta.

3. Jogiyanto, Hartono, 2013. Metode Penelitian Bisnis: Salah Kaprah dan Pengalaman-Pengalaman. Penerbit BPFE Yogyakarta. Yogyakarta.

4. Puspitasari, F. \& S.B. Santoso, 2013. Analisis Pengaruh Kualitas Layanan Inti Dan Kualitas Layanan Peripheral Terhadap Kepuasan Nasabah. Jurnal Studi Manajemen \& Organisasi Vol. 10, No. 2, Hal. 152-159.

5. Sugiyono. 2010. Metode Penelitian Administrasi: Pendekatan Kuantitatif, Kualitatif, dan R\&D. Alfabeta. Bandung.

6. Undang-Undang Republik Indonesia Nomor 5 Tahun 2014 tentang Aparatur Sipil Negara. 